\title{
Dimming Cellular Networks
}

\author{
David Tipper, Abdelmounaam Rezgui, Prashant Krishnamurthy, and Peera Pacharintanakul ${ }^{\dagger}$ \\ Graduate Telecommunications and Networking Program, \\ University of Pittsburgh, Pittsburgh, PA 15260, United States \\ Email: \{dtipper, arezgui, prashant, peerap\}@ sis.pitt.edu
}

\begin{abstract}
We propose a novel technique called dimming to improve the energy efficiency of cellular networks by reducing the capacity, services, and energy consumption of cells without turning off the cells. We define three basic methods to dim the network: coverage, frequency, and service dimming. We construct a multi-time period optimization problem to implement frequency dimming and extend it to implement both frequency and service dimming together. We illustrate the ability of dimming techniques to adapt the capacity and network services in proportion to the dynamic spatial and temporal load resulting in significant energy savings through numerical results for a sample network.
\end{abstract}

\section{INTRODUCTION}

Increasing worldwide energy demand, global warming concerns, and volatility in energy supplies and prices have necessitated improving the energy efficiency of ICT systems. Recent studies [1], [2] have shown that the largest elements of power consumption within the communications component of ICT, are access networks (wired and wireless). The fastest growing access network sector is cellular telephone networks. The ITU estimates that the number of cell phone subscribers hit 4.6 billion at the end of 2009 [3]. The need for energy efficiency in cellular networks has only been recognized very recently by industry, standards groups, and the research community [1], [2], [4] - [10]. Several studies have shown that less than $1 \%$ of the energy consumption in cellular networks is due to mobile user handsets while more than $99 \%$ of the energy consumption is due to the network itself [6]. A study by NTT DoCoMo, Japan's largest mobile telecom operator, found that the energy consumption ratio of terminals vs. network is about 1:150 [5]. Within the cellular network, base stations (BSs) are responsible for most of the energy consumption, consuming $50-90 \%$ of the network power depending on the network topology, BS configuration, radio technology and data rate used [1], [6], [7]. The growth in the number of BSs in the USA illustrates the scale of the increasing energy consumption in cellular networks. The number of cell sites in the USA increased from 183,689 in Dec., 2005 to 247,081 in Dec., 2009 [4]. With 3.47 radio base stations on average per cell site (due to co-location and sectorization), the number of radio base stations in the USA is closer to 750,000 , each of which consumes significant energy resources.

This research work is supported in part by a seed grant from the University of Pittsburgh Center for Energy.

${ }^{\dagger}$ The work of Peera Pacharintanakul was supported by a predoctoral fellowship from Office of the Provost, University of Pittsburgh.
Current cellular networks essentially operate in a $24 \times 7$ "always-on" mode consuming significant power even when not carrying traffic. The network traffic demand however has significant variations with time of year, time of day, geographic location, weather, social events, etc. In [10], the idea of powering down (sleeping) base stations with no significant traffic was proposed. However, for reasons such as meeting regulatory requirements, maintaining spatial coverage, synchronization, security and other concerns (described in Section III), sleeping may not be the right option. Thus, the challenge is how to reduce overall energy consumption in cellular networks while maintaining adequate coverage, quality of services, and reliability. We propose a novel approach to save energy in cellular networks with dimming techniques where-in one reduces the capacity/services and thus the energy consumption of a cell without turning off the cell entirely. BSs can be dimmed in three basic ways. First it is possible to just reduce the power of the $\mathrm{BS}$ and thereby the coverage area - we term this coverage dimming. Second, one can switch off radio frequencies (essentially cards in base stations) which will reduce the cell capacity, but saves energy by reducing the amount of transmit power and the energy needed for cooling requirements - we call this technique frequency dimming. Third, one can apply service dimming where high data rate services are disabled on a frequency. For a lower data rate, the transmit power is lower and cooling requirements are smaller thus saving energy.

In this paper, we develop network management techniques employing frequency and frequency \& service dimming to save energy in cellular access networks. We consider the spatial and temporal variation in the network traffic load to determine when and where to employ the dimming techniques which then dynamically size the network and provide only the capacity needed for the given load in a cell site. Given a set of traffic demands, we formulate an optimization problem to determine the amount of frequency and service dimming employed at each cell. Illustrative numerical results show that this can result in significant energy savings while still meeting the traffic demand. The rest of the paper in organized as follows. In Section 2, we describe a typical cellular network architecture and related literature on energy efficient management techniques. Section 3 contrasts sleeping with dimming. Section 4 presents our optimization based formulation of the proposed dimming techniques. Section 5 gives numerical results showing the amount of energy savings possible and 


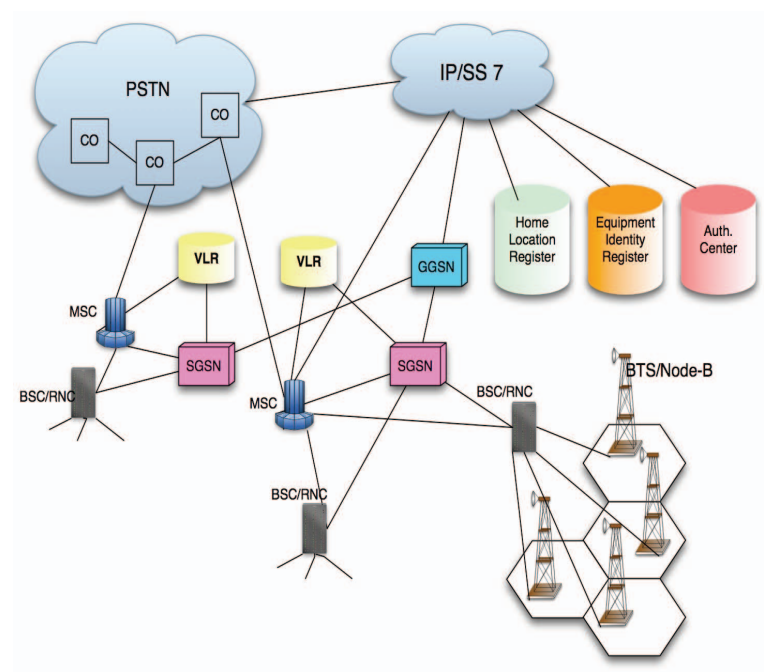

Fig. 1. A typical cellular network architecture

Section 6 presents our conclusions.

\section{BACKGROUND}

\section{A. Cellular Network Architecture and Traffic Characteristics}

We first present an overview discussion on the current cellular network architecture in the USA. The Federal Communication Commission regulates spectrum in the USA and has partitioned the country into 306 Metropolitan Service Areas (MSAs - which contain a city of at least 50,000 population) and 428 Rural Service Areas (RSAs) for geographic assignment of the licenses (map available at [11]). A generic 3G cellular mobile network architecture is illustrated in Figure 1 similar to a UMTS 3GPP Release 99 network. In UMTS the base station is termed a Node B and corresponds to a base station transceiver (BTS) in GSM. Similarly the UMTS Radio Network Controller (RNC) corresponds to a base station controller (BSC) in GSM and manages a group of base stations and performs radio level channel management and call handoff. The wireless link between a Node B and mobile is packet based using wideband CDMA (WCDMA) as the air interface standard. Each Serving GPRS support node (SGSN) detects and registers new mobile terminals in its service area, sends/receives data packets to/from the mobiles, and tracks the location of the mobiles within its service area. The gateway GPRS support node (GGSN) is the interface between the 3GPP network and external networks. The network may include mobile switching centers (MSC) for connecting to circuit switched networks. The MSC is connected both to transmission networks and to the signaling network. Associated with the signaling network and MSC and SGSN are databases to support user and service mobility (such as the Home Location Register (HLR) and Visitor Location Register (VLR)). Note that the number of cells and their location is normally determined to provide complete geographic coverage while satisfying the peak busy period traffic at that location.

Given the cellular network architecture shown in Figure 1 consider the network traffic load. While cellular voice and data traffic studies have not been widely published due to

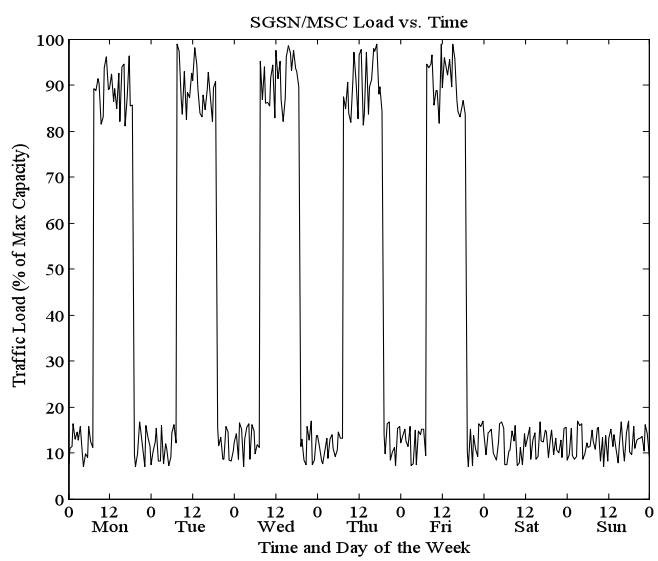

Fig. 2. Representative SGSN/MSC Load

privacy and operator concerns, a few have appeared and they clearly show temporal and spatial variations in the traffic [12] - [14]. Figure 2 shows representative aggregate data traffic at a SGSN/MSC for an MSA. Note that the traffic has a pronounced diurnal behavior which changes with day of the week and day of the year, with the traffic typically being much heavier on weekdays. At the level of cells, there are significant spatial variations of the magnitude and time when the peak busy period occurs (this has the potential for power savings during the day as well as at night). While the aggregate traffic would follow the behavior shown in Figure 2, the traffic at specific cells can vary greatly. Figure 3 shows a representative week of behavior for two cells, one in a downtown area (Cell 1) and the other in a shopping district (Cell 2). Notice that the traffic load in Cell 1 largely follows the work day. In contrast the traffic at Cell 2 which provides coverage to a shopping/entertainment district has a busy period much later in the day and skewed more towards the weekend as shown in Figure 3. One can see that the busy periods are non-coincident. We exploit this later in dimming cellular networks.
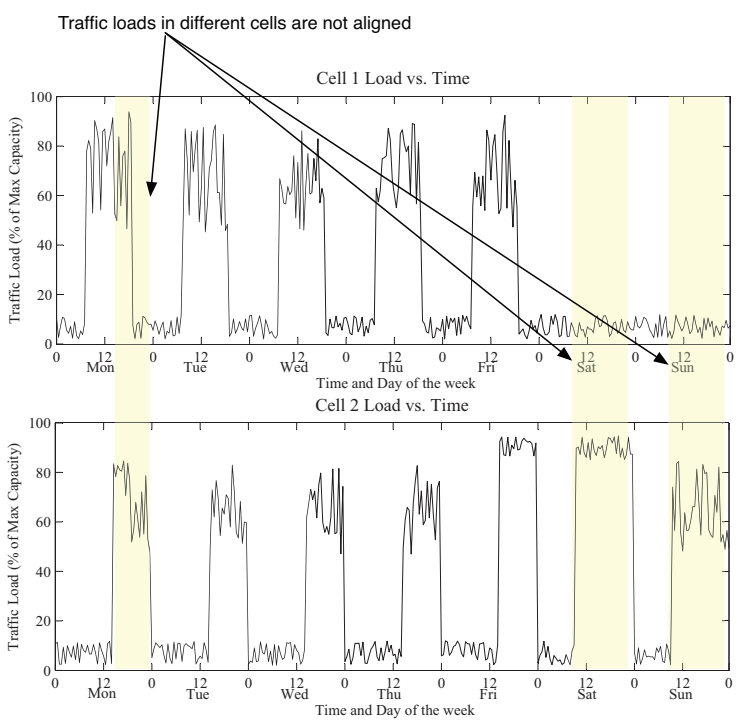

Fig. 3. Typical Load at Cells 1 and 2 for Various Times of Day 


\section{B. Related Literature}

Relatively few papers have appeared in the research literature on energy efficient cellular networks [1], [6] - [10] Many of the papers are essentially bringing awareness of the need for energy efficiency and the challenges in achieving it in cellular networks [1], [5]. Recently, the idea of powering down base stations with little or no traffic at night has been advocated [10]. Specifically, the authors look at the aggregate load on a cellular service area and based on a rather idealized analysis show that by turning off cells to track the time of day variations in the aggregate load operators can realize large savings (25-30\%) in energy. However, they do not consider the spatial distribution of traffic in turning off cells or the additional power needed to have neighboring cell expand their coverage to prevent dead spots in network coverage. A similar analysis of the wired backhaul portion of the network is given in [9] showing some possible energy savings by putting network components to sleep during low load periods. While the current literature shows the need for incorporating energy efficiency in cellular networks and provides some first cut analysis of the potential gains, it is lacking in several ways as discussed next.

\section{SLeEPING Vs DimMing}

\section{A. Drawbacks of Sleeping}

Shutting down BSs is not straightforward. First, in reality, the traffic has both spatial and time of day variations and other factors (weather, holidays, sporting and social events) affect the traffic load as well. Thus determining which cells to switch off, when and for how long is nontrivial and can adversely affect network performance. More importantly, it is difficult to envision network operators powering down cell sites or backhaul equipment entirely for a variety of reasons as discussed in the following. Turning off an entire cell will result in holes in network coverage, which can result in poor network performance (e.g., high handoff call dropping rate, high paging delays, lack of interworking across SGSN boundaries for Inter SGSN handoffs, etc.) even though the network load is low. Several papers have appeared in the literature looking at the effects of base station failures on cellular networks (e.g, [15], [16]). One can think of a scheduled base station power down equivalent to a failure. This previous work shows that the impact of loss of coverage is larger than the geographic area left uncovered. For example, the failure of a BSC in a GSM network knocking out a group of four adjacent cells (in a network of 100) results in the mean time to process a location update for the entire group of 100 cells to exceed (by a factor of 10) the recommended ITU benchmark value resulting in protocol timeouts. Further, the magnitude and duration of the impact of a loss of coverage (failure) depends on a complex set of factors including the location of the loss of coverage (e.g., center or edge of location update area, bordering another SGSN/MSC service area, etc.), shape of the uncovered area (e.g., adjacent or disjoint cells), user mobility patterns, and user behavior in attempting reconnection. In fact, the studies show that radio-level failure (e.g., loss of a BS) causes a large increase in transient congestion in the signaling network [15] due to paging, dropped handoff and location update signaling.

Another significant hurdle in turning off an entire cell site is that the loss of coverage can result in violations of regulatory requirements such as the USA E-911 localization requirements. The reader is referred to [17] for more details. Irrespective of whether AGPS (which employs assistance from BSs in addition to GPS) or TDOA (BS and network-based triangulation) positioning is used, the ability to simply power down BSs is impacted.

Additional considerations include issues as some common cellular equipment needs to maintain synchronization (e.g., cdma2000 1X-evdo base station equipment) which can be slow to reestablish after being powered down. Lastly, operators state the need to maintain security in their network necessitates keeping it powered up as they are unsure what is happening to their equipment when it is powered down entirely. Note that many of these comments apply to the backhaul as well as the wireless part of the network.

\section{B. Base Station Dimming}

As discussed above, the necessity of maintaining geographic coverage, backhaul interworking (e.g., Inter SGSN handoffs) and other considerations (e.g., E-911, synchronization, security) may significantly constrain the number of BSs that can be powered down. In light of these concerns, we propose a novel approach to energy efficient network management of cellular networks based on the use of dimming techniques where-in one reduces the capacity, services and energy consumption of a cell without turning off the cell entirely. Base stations can be dimmed in three basic ways, namely: (1) coverage dimming, (2) frequency dimming, and (3) service dimming.

Coverage dimming corresponds to reducing the geographic coverage area of a cell, which is achieved by reducing the base station operating power level on all deployed frequencies. For example, one can decide to not support soft handoff during low traffic load periods and reduce the overlapping coverage of cells. Note that the amount of power saved will be a complex function of the cell size, propagation environment, link power budget and cell configuration (antennas, location, etc). As such it may be difficult to plan exactly how much to reduce the power and still have power savings while providing adequate service and we do not consider this approach in detail in this paper. Secondly one can reduce the cell capacity and power use by switching off radio frequencies, we call this technique frequency dimming. This saves on both transmission power and heating/cooling requirements. Studies have indicated that up to $40 \%$ of the power consumption of a base station is in the heating and cooling. Thirdly, 3G (UMTS, cdma2000 1x-EVDO), 3.5G (HSPA) and 4G (LTE, WiMAX) networks provide a range of advanced mobile data services (e.g., MMS, streaming video, Internet access, video calls, multi-person gaming etc.) in addition to the $2 \mathrm{G}$ service offerings of SMS and voice. It is well known that the higher throughput of the advanced data services requires high power levels (i.e., larger $\left.E_{b} / N_{0}\right)$ if the same coverage footprint is maintained. Thus 
a power conserving technique for $3 \mathrm{G}, 3.5 \mathrm{G}$ and $4 \mathrm{G}$ cells is to consider service dimming of operational base stations to provide reduced services (e.g., $2 \mathrm{G}$ only). For example, at night along an Interstate highway a service provider can dim cells by disabling mobile data services and supporting only voice and SMS services.

Note that base station sleeping is a special case of coverage dimming (i.e., reduce power to zero) and frequency dimming (i.e., turn off all frequency channels).

\section{Optimal Dimming Models}

Here we consider both the frequency and service dimming techniques and develop a multi-time period optimization model formulation to determine the set of frequencies and services to be offered in each cell as a function of the load. We first formulate the frequency dimming only case and then the combined frequency and service dimming scenario.

\section{A. Frequency Dimming}

Consider a network composed of a set of $\mathrm{N}$ cells providing service in a specific geographic area (i.e., MSA or RSA). Let $F$ be the number of frequencies licensed for the service provider in the geographic service area. We define $F_{i}$ as the number of frequencies assigned to cell $i$. Let $P_{i}^{f}$ denote the power consumption of frequency $f$ in cell $i$ where $f \in\left\{1,2, \cdots, F_{i}\right\}$. Note that $P_{i}^{f}$ can be different for each frequency deployed in a cell and includes the heating and cooling power requirements as well as the transmission power needed. Let $C_{i}^{f}$ denote the capacity of frequency channel $f$ at cell $i$. We divide a day into a set $H_{j}$ of non-overlapping time periods $\left\{1,2, \cdots, H_{j}\right\}$ on day $j,\{j=1,2, \cdots, 365\}$. Note, that the time periods $h \in\left\{1,2, \cdots, H_{j}\right\}$ need not be equally long. For the sake of simplicity in the explanation, we formulate the problem for a single arbitrary day and drop the subscript for the day of the year. Let $W_{h}$ be the percentage of the day of time interval $h$ [i.e., (duration of time interval $h$ )/24]. Let $d_{i}^{h}$ be the traffic demand at cell $i$ during time interval $h \in\{1,2, \cdots, H\}$. We define $x_{i}^{f h}$ as a decision variable where $x_{i}^{f h}=1$ if frequency $f$ is used during time interval $h$ at cell $i$ and is equal to 0 otherwise. Given the definitions above, we seek to minimize the total power $P T$ used in the network while satisfying the demand for each time period $h$ in a day. This can be formulated as optimization problem P1 given below:

\section{Problem P1: Frequency dimming}

$$
\min _{x_{i}^{f h}} P T=\sum_{h=1}^{H} W_{h} \sum_{i=1}^{N} \sum_{f=1}^{F_{i}} P_{i}^{f} x_{i}^{f h}
$$

s.t.

$$
\begin{aligned}
\sum_{f=1}^{F_{i}} C_{i}^{f} x_{i}^{f h} & \geq d_{i}^{h} & \forall h, i \\
x_{i}^{f h} & \in\{0,1\} & \forall h, i, f
\end{aligned}
$$

The objective function (1) in problem P1 above, seeks to find the set of frequencies needed to result in the minimum power needed to carry all traffic demand at each cell in each time period in a day. Constraint set (2) ensures that the capacity allocated to a cell for a given time period is greater than the demand. Constraint set (3) ensures the zero or one nature of the decision variables. Note, that to ensure that at least one frequency is left on in each cell thereby maintaining coverage, requires that the $d_{i}^{h}$ be set greater than zero for all cells in all time periods.

\section{B. Service and Frequency Dimming}

Given the optimization problem (P1) above we wish to model the existence of service classes and consider the effects of reducing the services offered on an active frequency and thereby saving power. Let $s \in\{1,2, \cdots S\}$ be the set of services offered in the network. Service class $s=S$ represents the highest class of service and $s=1$ the lowest. For example an operator might offer a suite of service classes on the downlink such as, HSDPA 3.6 Mbps data $(s=4)$, UMTS $384 \mathrm{Kbps}$ data $(s=3)$, UMTS/GPRS $171 \mathrm{Kbps}$ data $(s=2)$, and UMTS/GSM voice $12.2 \mathrm{Kbps}(s=1)$. Typically offering a class of service implies that all the lower classes of service are offered as well. For example, if UMTS $384 \mathrm{Kbps}$ class $s=3$ is offered then $s=2$ and $s=1$ are also provided. Note, that if the coverage of the cell is to remain constant then higher rate data service requires more transmit power. Let $P_{i}^{f s}$ denote the power consumption of frequency $f$ in cell $i$ where $f \in\left\{1,2, \cdots, F_{i}\right\}$ when offering service class $s$ where $s \in\{1,2, \cdots S\}$. Then $P_{i}^{f S} \geq P_{i}^{f(S-1)} \geq \cdots P_{i}^{f 2} \geq P_{i}^{f 1}$. We define $y_{i}^{f s h}$ as a decision variable which is equal to 1 if frequency $f$ in cell $i$ provides service class $s$ during time interval $h$. The basic combined frequency and service dimming optimization problem can be formulated as optimization problem P2 below:

Problem P2: Service and frequency dimming

$$
\min _{y_{i}^{f s h}} P T=\sum_{h=1}^{H} W_{h} \sum_{i=1}^{N} \sum_{f=1}^{F_{i}} \sum_{s=1}^{S} P_{i}^{f s} y_{i}^{f s h}
$$

s.t.

$$
\begin{array}{cr}
\sum_{f=1}^{F_{i}} \sum_{s=1}^{S} C_{i}^{f} y_{i}^{f s h} \geq \sum_{s=1}^{S} d_{i}^{h s} & \forall h, i \\
\sum_{f=1}^{F_{i}} C_{i}^{f} y_{i}^{f S h} \geq d_{i}^{h S} & \forall h, i \\
\sum_{k=j}^{S} \sum_{f=1}^{F_{i}} C_{i}^{f} y_{i}^{f k h}-\sum_{r=j+1}^{S} d_{i}^{h r} \geq d_{i}^{h j} & j<S, \forall h, i \\
\sum_{s=1}^{S} y_{i}^{f s h} \leq 1 & \forall h, i, f \\
y_{i}^{f s h} & \in\{0,1\}
\end{array}
$$

The objective function (4) in problem P2 seeks to find the set of frequencies and services needed to result in the minimum power required to carry all traffic demand for each enabled service class at each cell in each time period. The constraint set (5) ensures that the capacity allocated for a cell 


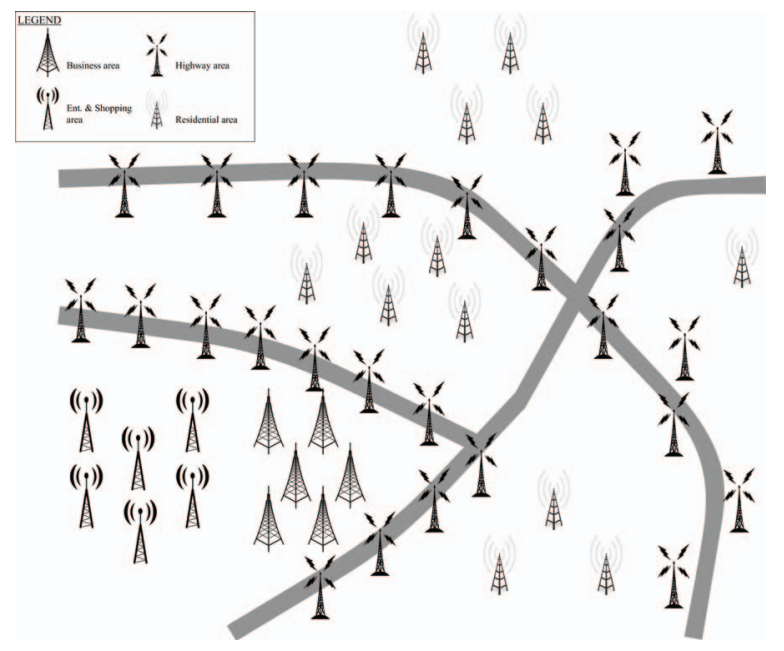

Fig. 4. Sample 50 Node Cellular Network

in a particular time period can carry all the traffic demand. Constraint sets (6) and (7) ensure that the enabled capacity for each service class can carry the traffic demand in that class. Specifically, constraint (6) requires that the capacity enabled for the highest service class in a cell exceed the highest service class demand in that time period. Similarly, constraint (7) assures that for the traffic demand in class $j$, the capacity enabled in the cell for classes $j$ and higher can carry the traffic for class $j$ and all higher classes. Constraints (8) and (9) require that only one service class $s$ be enabled for a frequency at a time and the decision variables are required to be zero or one. Thus, $y_{i}^{f s h}$ is 1 if for frequency $f$ service class $s$ is the highest service class enabled during period $h$ in cell $i$, and 0 otherwise. Recall that lower service classes can be supported by a higher one, e.g., only HSDPA needs to be enabled in order to support UMTS, GPRS, and GSM services.

Optimization problems (P1) and (P2) are integer programming problems that can be solved using standard techniques such as the branch and bound method. Typical values of $\mathrm{N}$ are 50-200 and we have solved this problem and similar ones for this problem size using commercial optimization solvers such as CPLEX. However scalability is an issue for larger problem sizes as integer programming problems have NP complexity and heuristics such as Lagrange relaxation, genetic algorithms, Tabu search, etc. may be needed for large cases.

\section{NumericAl RESUlts}

In this section, we present numerical results of solving the base station dimming models for a sample UMTS network illustrative of a small city. The base station layout consists of fifty cells $(N=50)$ as shown in Figure 4 . In the figure, the cells are classified into four categories based on their traffic profile and the primary societal use of the area covered, namely: (1) Business, (2) Entertainment and Shopping, (3) Highway, and (4) Residential. We divide a typical day into five time periods and the peak traffic load in each time period in terms of percentage of cell capacity is given in Table I.
TABLE I

PEAK TRAFFIC AS PERCENTAGE OF CELL CAPACITY

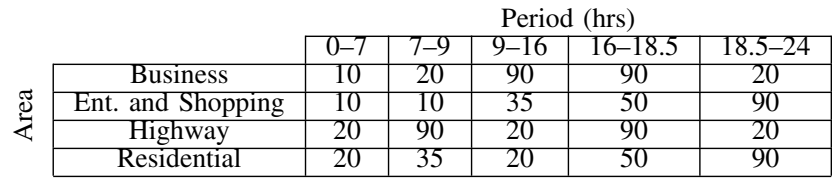

TABLE II

AVERAGE ENERGY SAVING PER BASE STATION TYPE

\begin{tabular}{|c|c|}
\hline Cell Type & Energy saving \\
\hline \hline Business & $48.33 \%$ \\
Ent. and Shopping & $51.67 \%$ \\
Highway & $65.00 \%$ \\
Residential & $44.79 \%$ \\
\hline
\end{tabular}

We assume the service provider has a spectrum license corresponding to five FDD $5 \mathrm{MHz}$ UMTS channels and deploys each frequency in every cell. Using typical data from UMTS manufacturers product data sheets we set the power consumption per frequency for HSDPA service in the business, entertainment and shopping, highway, and residential areas, as $165,165,210$, and $200 \mathrm{~W}$, respectively. Thus a cell in the residential area using all five frequencies would consume $1000 \mathrm{~W}$ of power. Numerical results for the dimming models are determined using the branch and bound algorithm implemented in the optimization solver CPLEX 9.1.

\section{A. Frequency Dimming}

First, we present numerical results for the frequency dimming model (P1). Figure 5 shows the energy consumption per time period per base station for each category of base station (business, entertainment, highway, residential) when frequency dimming is employed. One can clearly see from the figures that substantial energy savings are possible from frequency dimming. Computing the average daily energy savings in each traffic area we get the results shown in Table II. Notice that energy savings up to $65 \%$ are found in portions of the network.

\section{B. Frequency and Service Dimming}

To evaluate the effects of combined service and frequency dimming, we determined numerical results for the same fifty node network. We assumed four classes of service are provided at each cell (HSDPA data, UMTS data, GRPS data, UMTS/GSM voice). We approximated the energy savings per frequency by providing only lower class services by gathering data from UMTS base station manufacturers product data sheets. Specifically the power savings per frequency with the reduction in service from HSDPA to UMTS, GPRS, and GSM are $20 \%, 25 \%$, and $35 \%$, respectively.

The total load per category of base station follows the same time of day variations given in Table I. However, the mix of traffic among the four service classes for each time period also varies with time of day. In Table III, we show the traffic demand per service class as a percentage of the maximum capacity of the base station. The demands are given as a four-value tuple in decreasing order of service class (HSDPA, UMTS, GPRS, GSM). 

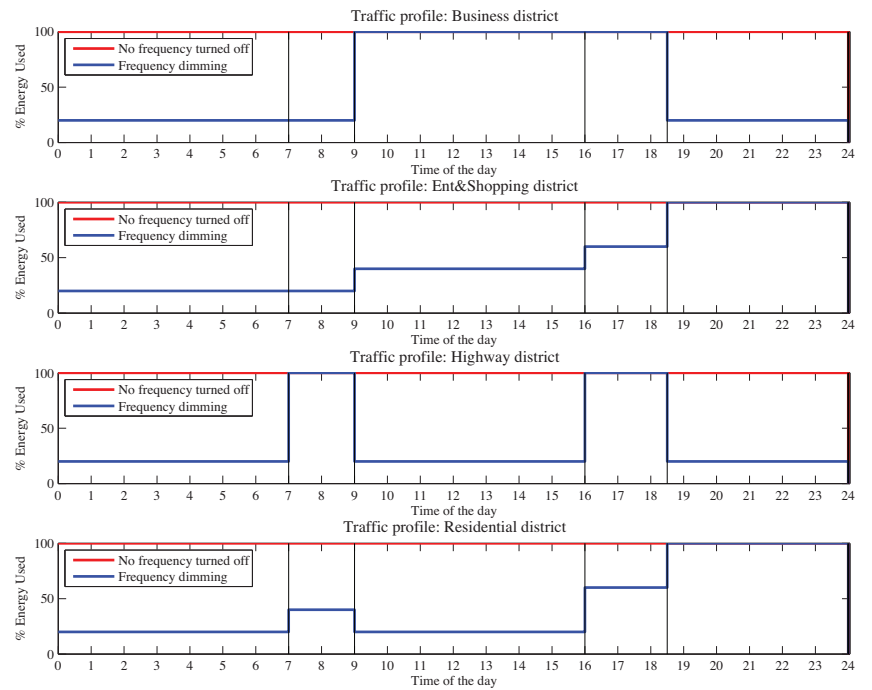

Fig. 5. Percentage of Maximum Power Consumption Vs. Time for Various Types of Cells with Frequency Dimming

TABLE III

Peak Traffic per Service Class for each Time Period as Percentage of the Cell Capacity

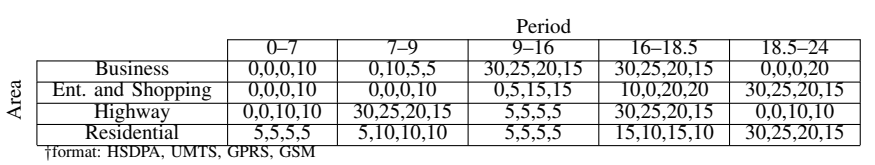

TABLE IV

AVERAGE ENERGY SAVING PER BASE STATION TYPE

\begin{tabular}{|c|c|}
\hline Cell Type & Energy saving \\
\hline \hline Business & $58.65 \%$ \\
Ent. and Shopping & $62.42 \%$ \\
Highway & $70.60 \%$ \\
Residential & $51.33 \%$ \\
\hline
\end{tabular}

Figure 6 shows the energy consumption per time period per base station for each category of base station (business, entertainment, highway, residential) when both frequency and service dimming options are utilized. Table IV lists the average daily energy savings for each base station type. One can see that service dimming provides additional energy savings on top of frequency dimming.

\section{CONCLUSION}

In this paper, we propose the novel idea of dimming cellular networks for energy savings. We explore frequency dimming which switches off certain frequency carriers in cells and service \& frequency dimming that disables certain high data rate services on enabled frequencies during periods where traffic demand is low. We formulate a framework for optimally dimming cells and show that up to $65 \%$ savings in average energy consumed in parts of a network is possible with frequency dimming and up to $70 \%$ savings in average energy consumed in parts of a network is possible with both frequency and service dimming.
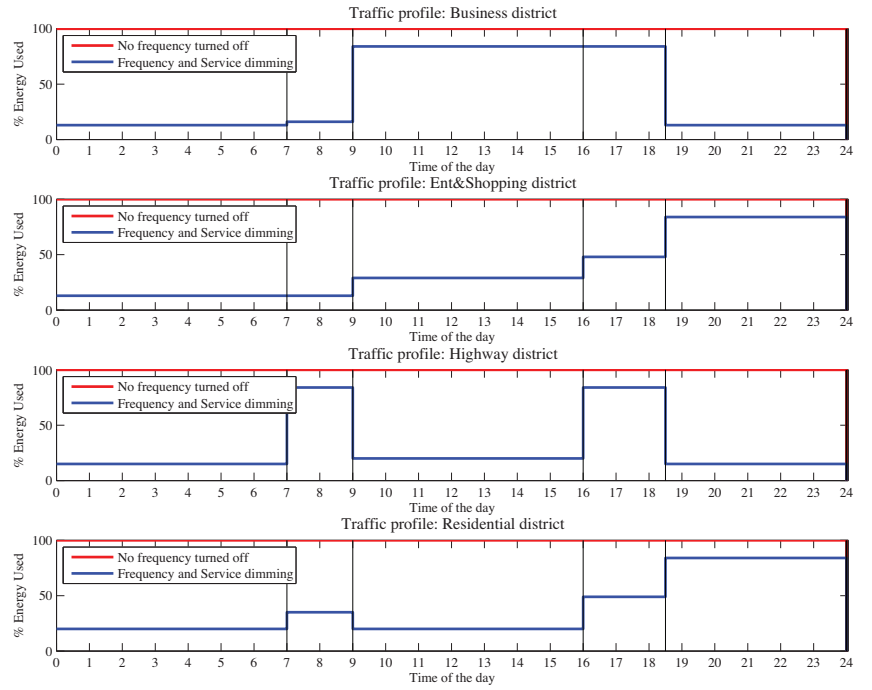

Fig. 6. Percentage of Maximum Power Consumption Vs. Time for Various Types of Cells with Frequency and Service Dimming

\section{ACKNOWLEDGMENT}

The authors would like to thank Mr. Tae-hoon Kim for his help with some of the illustrations in this paper.

\section{REFERENCES}

[1] G. Fettweis and E. Zimmermann, "ICT energy consumption - trends and challenges," The 11th International Symposium on Wireless Personal Multimedia Communications (WPMC), 2008.

[2] ITU and climate change. [Online]. Available: http://www.itu.int/themes/climate/index.html

[3] ITU, The World in 2009: ICT Facts and Figures. Geneva, Switzerland: ITU Telecom World, October 2009.

[4] CTIA: The wireless association. [Online]. Available: http://www.ctia.org

[5] "Green mobile networks and base stations: Strategies, scenarios and forecasts 2009-2014," Juniper Report, Tech. Rep., July 2009.

[6] M. Etoh, T. Ohya, and Y. Nakayama, "Energy consumption issues on mobile network systems," International Symposium on Applications and the Internet (SAINT), 2008.

[7] D. Lister, "An operator's view of green radio, keynote speech," in Proc. IEEE GreenCom, June 2009.

[8] "Green communications," ICC 2009 Panel P4, IEEE International Communications Conference, June 2009.

[9] L. Chiaraviglio, M. Mellia, and F. Neri, "Energy-aware umts core network design," The 11th International Symposium on Wireless Personal Multimedia Communications (WPMC), 2008.

[10] M. Marsan, L. Chiaraviglio, D. Ciullo, and M. Meo, "Optimal energy savings in cellular access networks," in Proc.IEEE GreenCom, Jun 2009.

[11] FCC wireless telecommunications bureau cellular market areas. [Online]. Available: http://wireless.fcc.gov/auctions/data/maps/CMA.pdf

[12] E. Halepovic, C. Williamson, and M. Ghaderi, "Wireless data traffic: A decade of change," IEEE Network, vol. 23, no. 2, March 2009.

[13] T. Winter, U. Turke, and M. Koonert, "A generic approach for including live measurements and traffic forecasts in the generation of realistic traffic scenarios in mobile radio networks," ACM International Workshop on Modeling Analysis and Simulation of Wireless and Mobile Systems (MSWiM 2004), Oct 2004.

[14] C. Williamson, E. Halepovic, H. Sun, and Y. Wu, "Characterization of CDMA2000 cellular data network traffic," Proc. IEEE LCN, Nov. 2005.

[15] D. Tipper, C. Charnsripinyo, H. Shin, and T. Dahlberg, "Survivability analysis for mobile cellular networks," CNDS, 2002.

[16] A. P. Snow, U. Varshney, and A. D. Malloy, "Reliability and survivability of wireless and mobile networks," IEEE Computer, vol. 33, pp. $49-55,2000$.

[17] Y. Zhao, "Standardization of mobile phone positioning for $3 \mathrm{~g}$ systems," IEEE Communications Magazine, pp. 108-116, 2002. 